\title{
The Athena x-ray optics development and accommodation
}

\section{Marcos Bavdaz, Eric Wille, Mark Ayre, Ivo Ferreira, Brian Shortt, et al.}

Marcos Bavdaz, Eric Wille, Mark Ayre, Ivo Ferreira, Brian Shortt, Sebastiaan Fransen, Mark Millinger, Maximilien J. Collon, Giuseppe Vacanti, M. Barrière, Boris Landgraf, Mark O. Riekerink, Jeroen Haneveld, Ronald Start, Coen van Baren, Desiree Della Monica Ferreira, Sonny Massahi, Sara Svendsen, Finn Christensen, Michael Krumrey, Evelyn Handick, Vadim Burwitz, Miranda J. Bradshaw, Giovanni Pareschi, Giuseppe Valsecchi, Dervis Vernani, Geeta Kailla, William Mundon, Gavin Phillips, Jakob Schneider, Tapio Korhonen, Alejandro Sanchez, Dominique Heinis, Massimiliano Tordi, Richard Willingale, "The Athena x-ray optics development and accommodation," Proc. SPIE 11852, International Conference on Space Optics - ICSO 2020, 1185220 (11 June 2021); doi: 10.1117/12.2599341 SPIE. Event: International Conference on Space Optics - ICSO 2021, 2021, Online 


\section{International Conference on Space Optics-ICSO 2020}

Virtual Conference

30 March-2 April 2021

Edited by Bruno Cugny, Zoran Sodnik, and Nikos Karafolas
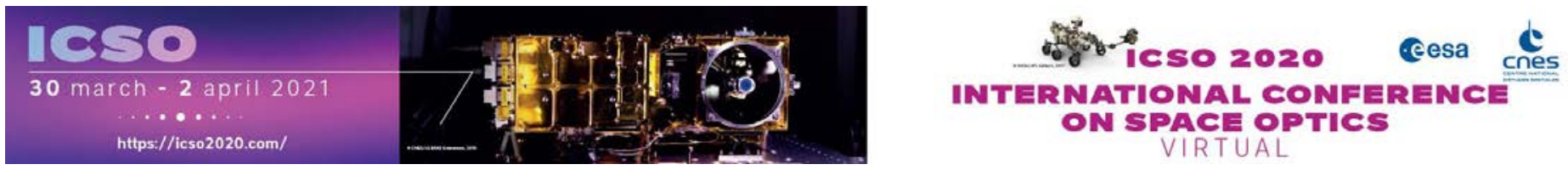

\section{The Athena x-ray optics development and accommodation}

\section{Cesa isopueatans lecnes}

International Conference on Space Optics - ICSO 2020, edited by Bruno Cugny, Zoran Sodnik, Nikos Karafolas, Proc. of SPIE Vol. 11852, 1185220 - (c) 2021 ESA and CNES

CCC code: $0277-786 \mathrm{X} / 21 / \$ 21 \cdot$ doi: $10.1117 / 12.2599341$ 


\title{
The ATHENA X-ray Optics Development and Accommodation
}

\author{
Marcos Bavdaz $^{1}$, Eric Wille ${ }^{1}$, Mark Ayre ${ }^{1}$, Ivo Ferreira ${ }^{1}$, Brian Shortt ${ }^{1}$, Sebastiaan Fransen ${ }^{1}$, Mark \\ Millinger ${ }^{1}$, Maximilien J. Collon ${ }^{2}$, Giuseppe Vacanti ${ }^{2}$, Nicolas M. Barrière ${ }^{2}$, Boris Landgraf ${ }^{2}$, Mark \\ Olde Riekerink ${ }^{3}$, Jeroen Haneveld ${ }^{3}$, Ronald Start ${ }^{3}$, Coen van Baren ${ }^{4}$, Desiree Della Monica Ferreira ${ }^{5}$, \\ Sonny Massahi ${ }^{5}$, Sara Svendsen ${ }^{5}$, Finn Christensen ${ }^{5}$, Michael Krumrey ${ }^{6}$, Evelyn Handick 6 , Vadim \\ Burwitz 7 , Miranda Bradshaw ${ }^{7}$, Giovanni Pareschi $^{8}$, Giuseppe Valsecchi ${ }^{9}$, Dervis Vernani ${ }^{9}$, Geeta \\ Kailla11, William Mundon ${ }^{11}$, Gavin Phillips ${ }^{11}$, Jakob Schneider ${ }^{12}$, Tapio Korhonen ${ }^{13}$, Alejandro \\ Sanchez ${ }^{14}$, Dominique Heinis ${ }^{14}$, Massimiliano Tordi $^{15}$, Richard Willingale ${ }^{16}$

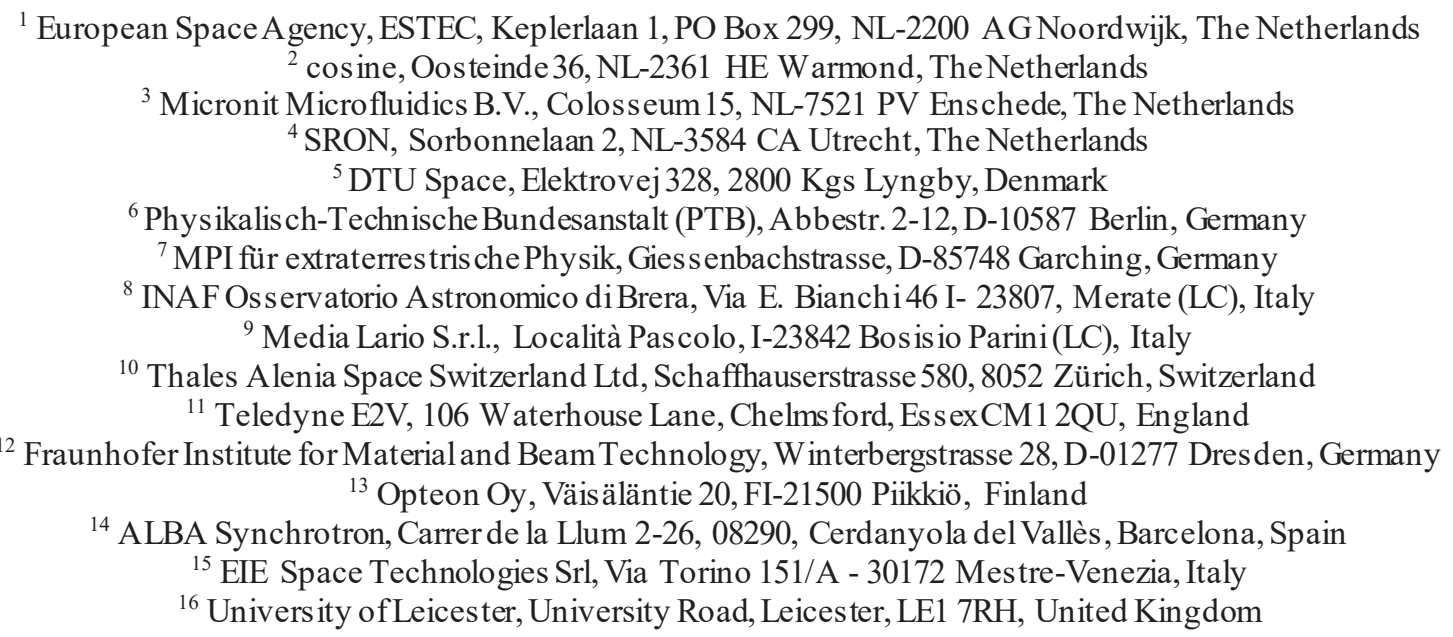

\begin{abstract}
The ATHENA mission, under study and preparation by ESA as its second Large-class science mis sion, requires the largest X-ray optics ever flown, building on a novel optics technology based on mono crystalline silicon. Referred to as Silicon Pore Optics technology (SPO), the optics is highly modular and benefits from technology s pin-in from the semiconductor industry. The telescope aperture of about 2.5 meters is populated by 600 mirror modules, accurately co aligned to produce a common focus.
\end{abstract}

The development of the SPO technology is a joint effort by European industrial and research entities, working together to address the challenges to demonstrate the imaging performance, robustness and efficient series production of the ATHENA optics. A technology developmentplan was es tablished and is being regularly updated to reflect the latest developments, and is fully funded by the ESA technology development programmes. An ind ustrial consortium was formed to ensure coherence of the individual technology development activities.

The SPO technology uses precision machined mirror plates produced using the latest gen eration top quality 12 in ch silicon wafers, which are assembled into rugged stacks. The surfaces of the mirror plates and the integral support structure is such, that no glue is required to join the individual mirror plates. Once accurately aligned with res pect to each other, the surfaces of the mirror plates merge in a physical bonding process. The resultant SPO mirror modules are therefore very accurate and stable and can sustain the harsh conditions encountered during launch and are able to tolerate the space environment expected during operations. 
The accommodation of the ATHENA telescope is also innovative, relying on a hexapod mechanismto align the optics to the selected detector ins truments located in the focal plane. Systems tudies are complemented by dedicated technology development activities to demonstrate the capabilities before the adoption of the ATHENA mission.

Keywords: X-ray optics, X-ray astronomy, ATHENA, Silicon Pore Optics, X-ray telescopes, X-ray testing, Technology preparation

\section{NOVEL OPTICS FOR THE NEXT GENERATION X-RAY SPACE TELESCOPE}

ATHENA (Advanced Teles cope for High-ENergy Astrophysics) [1] is the second Large Clas s ESA Science mis sion selected for flight in the early 2030s, aiming at studying the hot and energetic Universe at X-ray energies. With its ambitious sciencegoals it poses very demanding requirements on theX-ray optics and the payload detector instru ments $[2,3]$.

No X-ray optics technology flown to date can satisfy the needs of ATHENA. Some X-ray optics are light en ough and could achieve the large effective area, but would not be able to achieve the required angular resolution. Other technologies achieve excellent angular resolution, but would require thick mirrors, resulting in prohibitive mass.

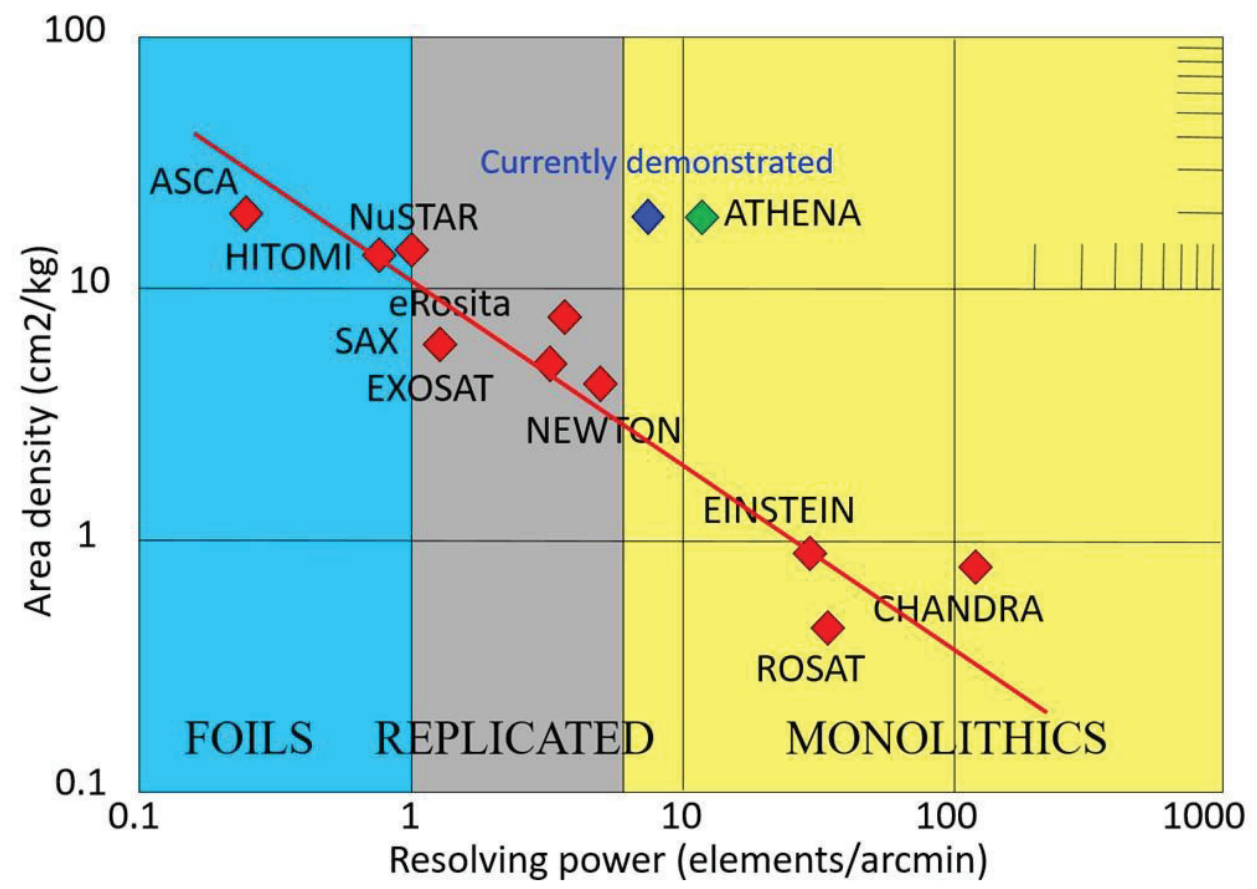

Figure 1: ATHENA require a radically new optics technology. It is interesting to note, that the X-ray optics flown to date show a correlation between the resolving power achieved (i.e. the number of angular resolution elements provided per arc minute) and the required area density (i.e. the effective area provided per $\mathrm{kg}$ of optics). This correlation extends over the different types of X-ray optics technologies used: (1) foil based optics (light blue: as used e.g. for HITOMI [4], or slumped glass, as for NuST AR [5]), (2) replicated optics (light grey, e.g. NEWTON [6]), and (3) monolithic optics (yellow, e.g. CHANDRA [7]). AT HENA requires very large optics (about $2.5 \mathrm{~m}$ diameter) with a low mass, and at the same time it has to provide a good angular resolution (see green diamond). Effectively the ATHENA optics technology has to be as light as foil optics, but perform like the monolithic optics. The Silicon Pore Optics (SPO) technology delivers the required performance for ATHENA: the blue diam ond in the graph indicated the performance demonstrated today. The SPO technology is being further improved to match the ATHENA needs. 
Figure 1 illustrates the challenge. Plotting the effective area provided per mass unit $\left(\mathrm{cm}^{2} / \mathrm{kg}\right)$ as function of the ang ular resolution expressed as angular resolution elements per arc minute, the X-ray missions flown to date show a clear correlation. Missions like HITOMI [4], relying on foil based optics, or on slumped glass like NuSTAR [5], missions like NEWTON [6] using replicated optics, and missions like CHANDRA [7] relying on monolithic optics all follow this correlation line, despite being so different in terms of X-ray optics technology. Nature is telling us a mes sagehere.

ATHENA requires an unprecedented combination of low mass, large effective area and good an gular resolution. As indicated in figure 1 by a green diamond, these requirements are significantly off the correlation line populated by existing missions.

Evidently ATHENA requires a novel type ofX-ray optics, which exceed the performance of existing technologies.

Silicon Pore Optics (SPO) [8-31] was invented in order to enable missions like ATHENA. With its pore structure it requires the optical elements to span much shorter dis tances between support points, and therefore achieves to comb ine thin (and therefore low mass) mirrors with good figure accuracy, resulting in good angular resolution.

The SPO technology has already demonstrated that it uniquely combines mass efficiency and imaging performan ce in a way no other mission succeeded to date, as indicated by the blue diamond in figure 2 . The evolution of the SPO technology is ongoing, moving the blue diamond to the right, with the aim to reach the position of the green diamond by the time of adoption of the ATHENA mis sion, expected in 2022.

\section{MODULAR SILICON PORE OPTICS}

In order to make it possible to build the ATHENA X-ray optics, the optics must be modular. Small optical elements are required, since only these can be produced effectively within the schedule and cost constraints. The production of the ATHENA optics has to be automatized as far as possible, to ensure constant quality, fast production and low cost.

As shown in figure 2, ATHENA spacecraft on first glace is quite similar to NEWTON or CHANDRA, albeit being larger, with its $12 \mathrm{~m}$ focal length. The payload ins truments are significantly larger and more complex, requiring elaborate cooling and EMC protection. The optics is substantially larger, with an optical diameter of about $2.5 \mathrm{~m}$. The optics consists of a metallic structure populated by 600 SPO mirror modules and holding metrology equipment inclu ding s tar trackers and alignment tools.

Activities have started to demonstrate the production of the complete full size ATHENA Mirror A ssembly demonstrators (two parallel contracts with the prospective possible ATHENA primes). These will serve to confirm the en viro n mental requirements to be imposed onto the SPO mirror modules, and to demonstrate the feasibility to manufacture such a large $\mathrm{X}$-ray optics within specifications.

The 600 mirror modules must be accurately co-aligned to focus onto the same spot in the focal place. The required AIT (Assembly Integration and Testing) process has been developed [32-34] and demonstrated to exceed the required performance.

The SPO mirror modules development started more than a decade ago, and has achieved a high level of tech nological readiness. Production equipment is in place to produce thousands of mirror plates and dozens of mirror modules per year. This was made possible by the implementation of robotic equipment and industrial production tools already in the development phase. 


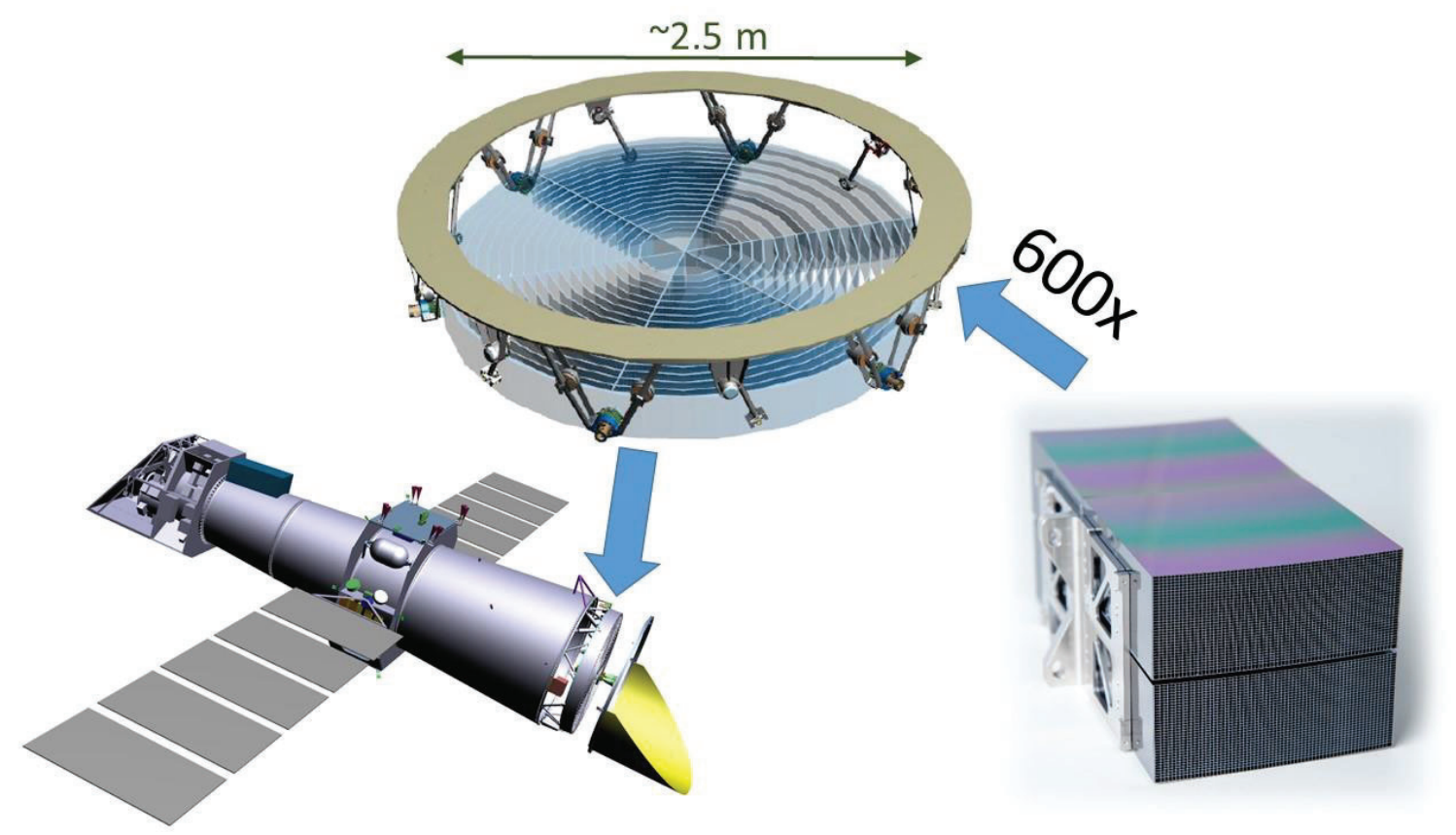

Figure 2: The ATHENA optics is modular, with 600 mirror modules populating its aperture. Silicon Pore Optics (SPO) can be mass produced at a competitive cost, relying on robotic production and mass produced commercial mirror elements. The SPO mirror modules are robust and compact, each effectively representing a complete imaging X-ray optical unit.

A large number of technology development activities were initiated and implemented by ESA, contracting key players in industry and res earch institutions. Expertise was brought together in focused activities, each having a clear objectiveand measurable targets. The cooperative spirit was required within each of the technology development activities, and a close coordination between the many activities was mandatory.

A Technology Development Plan (TDP) was elaborated (figure 3) and is kept updated to consider technical and programmatic evolution. All activities are fully funded and led by ESA.

The activities cover three areas: (1) the SPO mirror modules including mirror plate production and coatings, (2) the mirror assembly module including the optical bench and the AIT process, and (3) nu merical simulations, study of interaction with particles (mainly protons and micrometeoroids), and the preparation of the required test and verification facilities.

All major activities have been started and are progressing in their implementation. The extension of the main SPO mirror module activity is planned for mid-2021 (dashed red box).

The technology development activities will be concluded by the time of the Mission Adoption Review (MAR), expected for 2022. At the MAR the results of the ATHENA systemstudies [35] and technology activities will undergo a thorough review, in order to establish the readiness of the mission to pass into the implementation phase. The feasibility of the mission will have to be demonstrated in technical and programmatic aspects.

For the optics at MAR the performance will have to have been demonstrated in the relevant environment [36], i.e. the launch loads and operation in the space environment for the duration of the mis sion will have to be demonstrated, as well as the compatibility with the schedule, res ources (mainly mass), and cost constraints. 


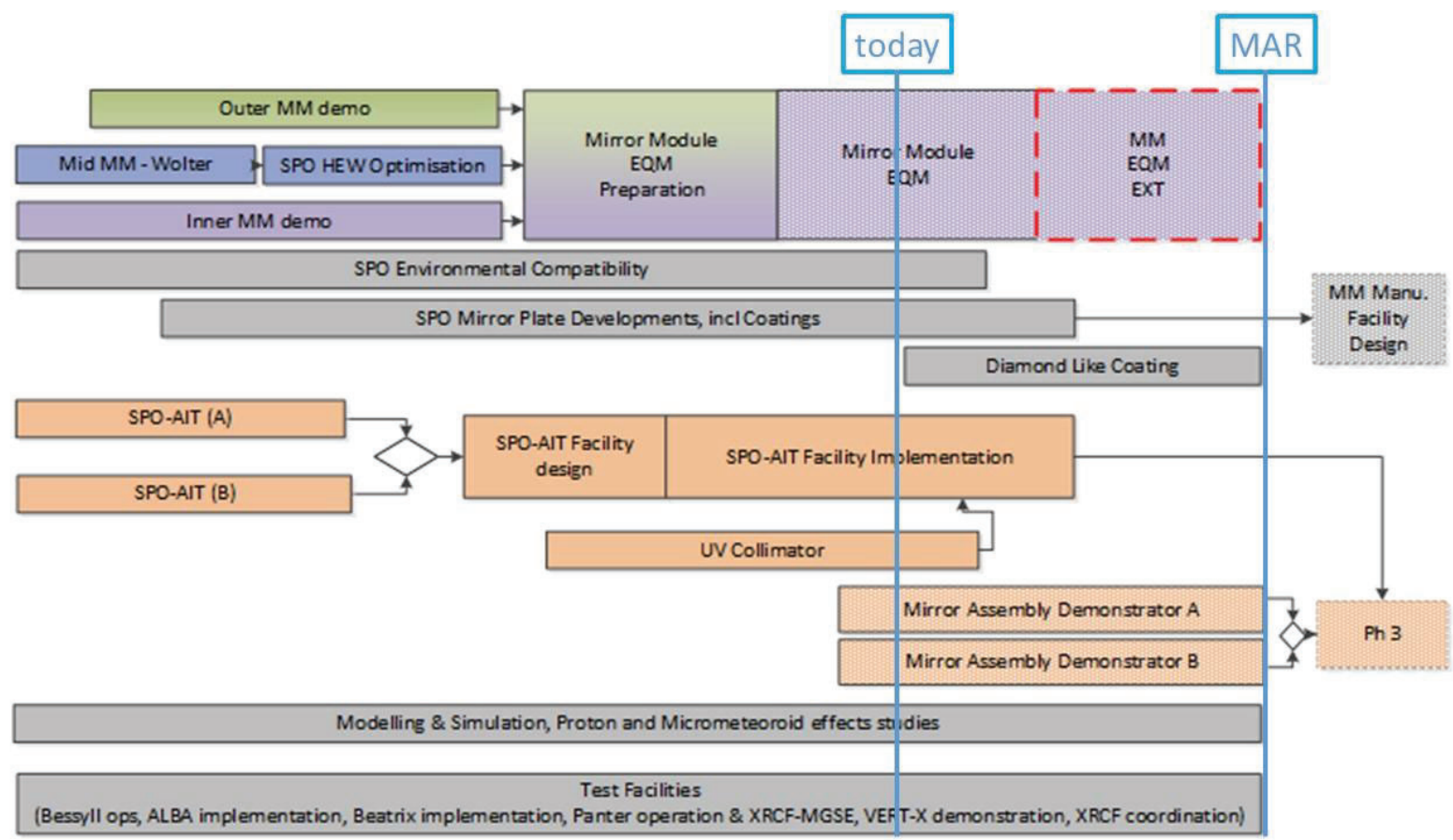

Figure 3: A comprehensive technology plan was elaborated and is being evolved and implemented by a consortium of industry and institutions. The development programme is fully funded and led by the European Space Agency (ESA). The group of activities indicated in the top of the graph regard the development of the SPO mirror modules, the center cluster of activities (in dicat ed in orange) regard the assembly of hundreds of mirror modules into a single optical assembly (the ATHENA Mirror Assembly), and the group of activities indicated in the bottom of the graph complement the development activities, addressing numerical simulations, test facilities and particle effects.

The performance of the SPO optics is continuously improving, as shown in figure 4, plotting the angular res olution of the SPO optics over time. The results shown are X-ray measurements of the optics in double reflection, meas ured at the Physikalis ch-Technische Bundesanstalt (PTB) laboratory of the BESSY-II synchrotron facility [37,38].

The primary and secondary stacks are mounted in tandem, as required for the Wolter-I config uration. Two cases are shown: the first 10 mirror plate pairs on the left, and the first 20 plate pairs on the right. The percentages indicated in the figure refer to the contiguous fraction of the optics width contributing to the value shown. In all cas es the complete height is measured (i.e. 10 and 20 plates res pectively).

The potential of the SPO technology is evident from the 10 plate result: $10 \%$ of the area is already outperforming the requirement, featuring a 4.2" HEW. The increas e of the area fraction with high performance is the target of the on going activities.

The complete area, i.e. 100\% of the width and measuring all stacked plates shows a HEW of 9.4" HEW. Note that this is already substantially better than the NEWTON optics, but at a much reduced mas s and cost per effectivearea.

The further improvement of the angular resolution of the SPO modules is the focus of the main ongoing tech nology development activity, and several augmentations of the tooling and mirror plates are under work. 
It is important to note, that the measured samples were made using largely automated equipment, therefore giving very repeatable results. The production method will essentially remain the same when moving into the implementation phase, and is not a one-off attempt to produce the best possible mirror module.

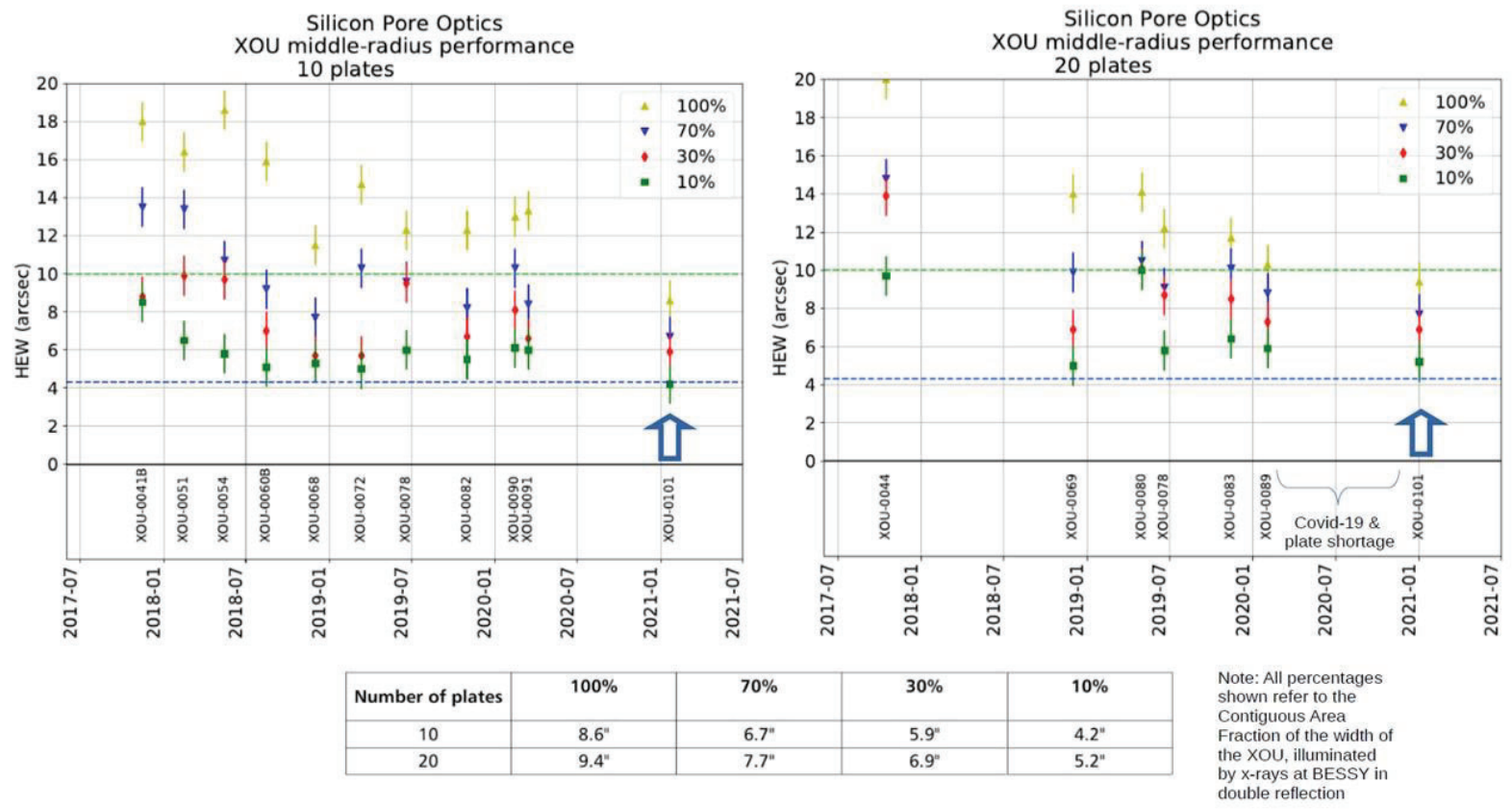

Figure 4: The SPO technology is evolving rapidly. The graphs above show the improvement of the angular resolution (Half Energy Width, HEW) obtained with SPO optics over time. XOUs are X-ray Optical Units consisting of the two required optical elements to provide true imaging (these are the primary and secondary reflectors of a Wolter type I system). Each optical elem ent consist of a stack of co-aligned mirror plates. The data shown integrates the performance of the lower 10 and 20 plates, respectively, and the percentages indicated refer to the contiguous area fraction measured (i.e the width of the area measured). These measurements were performed at the Physikalisch-Technische Bundesanstalt (PTB) laboratory of the BESSY-II facilit y in Berlin, in double reflection. Full area performance is already at 9.4" HEW, and smaller areas outperform the required 4.3" HEW.

\section{100.000 MIRRORS, 600 MODULES, ONE MIRROR ASSEMBLY}

The development of the ATHENA optics is undertaken with a simultaneous development of the tooling and equip ment required to manufacture, as semble, integrate, test and verify theoptics. This is an essential component of demonstrating the ATHENA optics technology readiness.

The ATHENA telescope with require the production of about 100.000 flight quality mirror plates. The majority will be used in the flight optics, others are required to populate the engineering and qualification mode ls, or will end up as flight spares.

Considering this large number of mirror plates to be produced, it was seen as mandatory to establish two man u factu res (Micronit in The Netherlands and Teledyne E2V in the United Kingdom) able to produce the required mirror plates. The approach being that each single manufacturer should be able to produce the complete set of mirror plates for ATHENA, but expecting to be required to deliver about half. Any potential problems with the mirror plate pro duction du ring the flight programme are thereby significantly de-risked. 
The mirror plates require many steps in their production, employing automated equipment as far as possible. The starting material for the mirror plate production is available on the semiconductor market: high quality single cry stal $300 \mathrm{~mm}$ diameter wafers, with both sides highly polis hed to the required X-ray super-polish finish. These surfaces are as s mo oth as required for the bonding operation when stacking the plates.

Figure 5 shows some of the equipment used for the mirror plate production. One of the work horses is the dicing machine shown on the bottom right, available at each of the mirror plate manufacturers. These machines work autonomously, ingesting pris tine wafers and producing the ribbed structure required on theplates, as well as dicing them to the precise dimensions required.

One of the large advantages of the SPO technology is the accessibility of the reflective mirror surface right up to the stacking operation. This makes it possible to use elaborate cleaning and coating processes to improve the reflectivity and therefore the effective area of the optics.
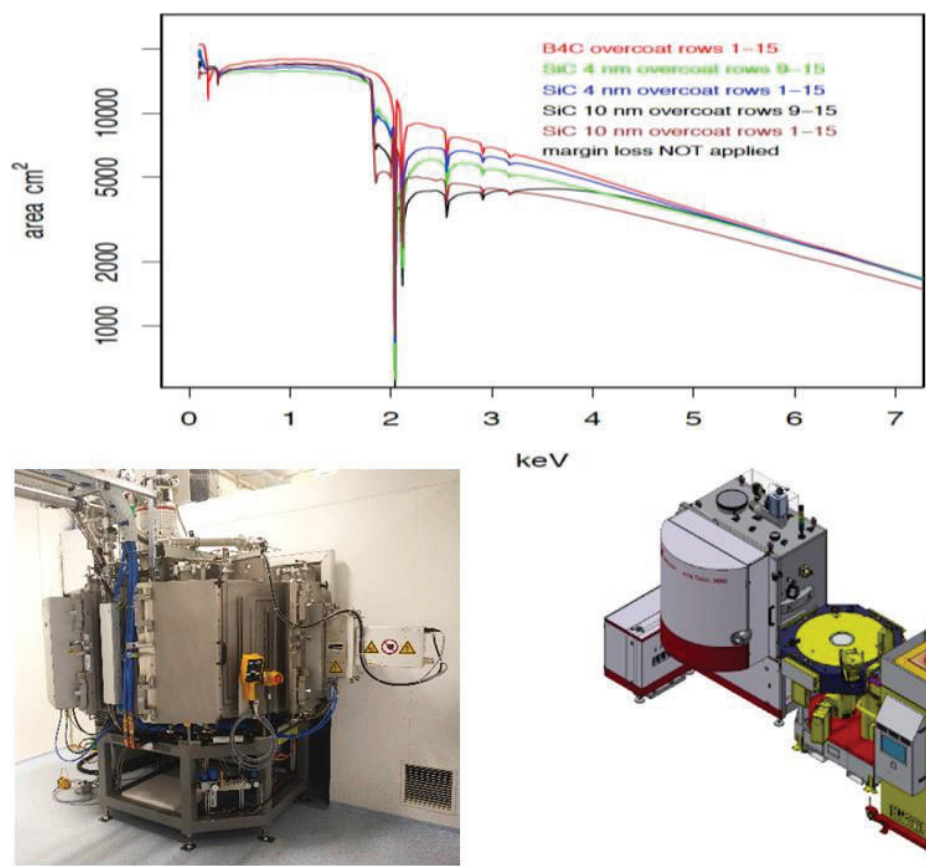

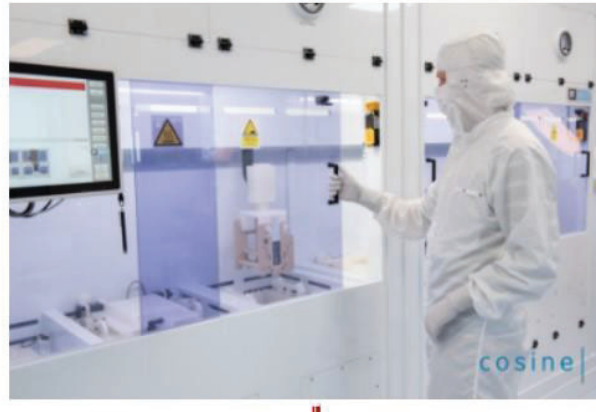

II

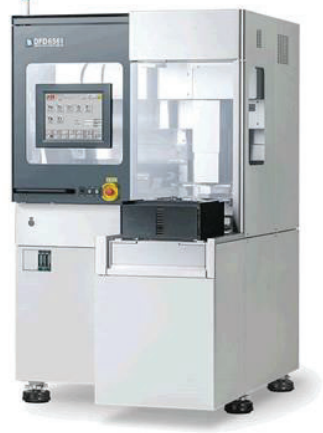

Figure 5: About 100.000 mirror plates will be required for the implementation of the ATHENA optics (including qualification and spare mirrors). This demands an industrial approach to the production of mirror plates. Currently about 2.000 mirror plates are already being produced per year, to feed the SPO mirror module and other developments. Industrial equipment as shown on the two bottom left photos is used to manufacture the mirror plates. Coatings can be sputtered onto the SPO mirror plates, and are an important element contributing to the performance of the ATHENA optics. In the top left a study of different coat ing option s is shown. The coatings are applied using commercial equipment: the machine shown on the bottom left was already installed at the SPO mirror module manufacturer, cosine, and is able to coat all the required 100.000 mirror plates in the allocated two years. An Ion Beam Figuring (IBF) machine is currently being procured, and will allow the further improvement of the mirror plates, allowing also for corrections to me made on individual plates.

On the top left a snapshot of a coating optimis ation study is provided, led by DTU in Denmark, building of their large heritage in making X-ray mirror coatings. Equally important is the ability to reproducibly apply the required coating onto the mirror surfaces, for $10 \mathrm{~s}$ of thousands of mirror plates, within the short time interval permitted by the mission 
implementation schedule. A suitable industrial coating machine, shown on the bottomleft of figure 5 , was there fore ins talled at the cosine premises. This single machine has a throughput sufficient to coat all the ATHENA mirror plates in the allotted time.

The SPO mirror plates are processed by both plate manufacturers in batches, e.g. in automated wet benches as shown in figure 5 top right, reducing the cost and allowing reference plates to be pulled from the production without much affecting the production yield. This type of equipment has also recently been installed at cosine to remove the lithographic resist, provide additional cleaning, and plate bonding preparation.

An Ion BeamFiguring (IBF) machine is being manufactured (it will be a new modelallowing automatic processing of $300 \mathrm{~mm}$ wafers ) and will be commis sioned at cosine in 2021. This equipment will allow the further improvement of the mirror plate figure, and make it possible to produce 'reset' mirror plates to minimize stack-up errors.

After production and quality acceptance each mirror plate has to me mounted, ensuring it is formed into the right $\mathrm{s}$ hape, and securing its figure for the duration of the mission.

Figure 6 shows the robotic stacking machine used to mount the mirror plates (top left). To ensure the cleanliness and repeatability of the process, the operation is highly procedural and defined in softwa re routines. The operators are located remotely and control the process forma dedicated control room(top, middle).
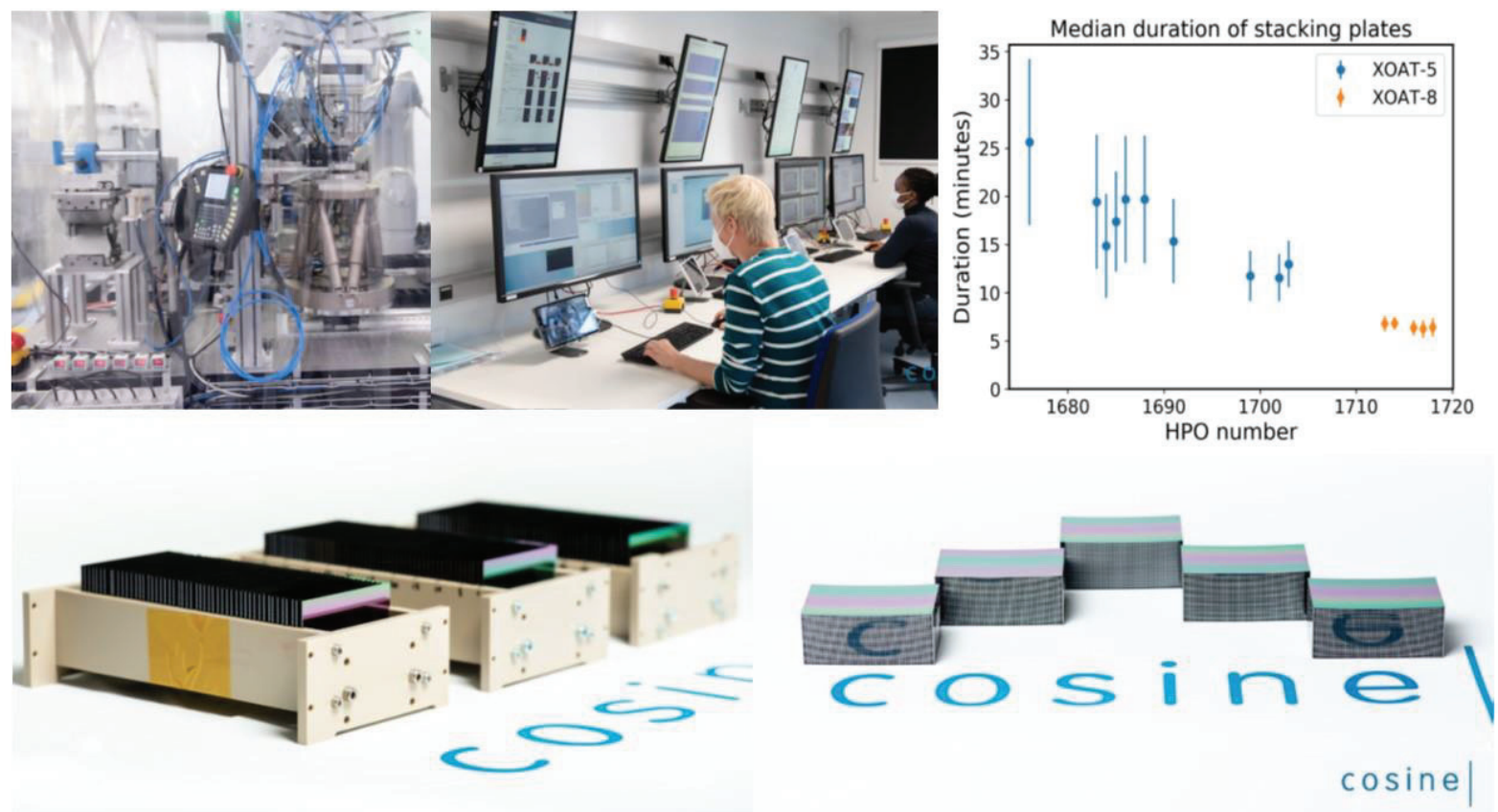

Figure 6: The SPO mirror plates are assembled into mirror stacks. This operation is performed robotically at cosine, ensuring the cleanliness and repeatability required for the operation. Complex equipment is being employed, controlled remotely by operato rs, to very efficiently produce the mirror stacks. The time required to mount a single mirror plate has been constantly decreasin g, and is already adequate for the production of the ATHENA telescope: only about 7 minutes are required to mount a mirror plate!

This stacking process is a critical step in the production of the SPO mirror modules and required attention to many details. The process must formeach mirror plate into the correct shape, deposit it onto the growing mirror plate s tack, and ensure that the correct figure is achieved over the complete area. 
Despite this complexity, only a few minutes will be available to mount each mirror plate in the flight programme. As shown in figure 6 top right, currently about 7 minutes are required to mount a mirror plate, which is fast enough already.

SPO mirror modules are being developed for three radii simultaneously. Indeed fours stackin g robots have been built up, ensuring that all required developments can occur in parallel. In figure 7, at the right, computer renderings of the outer, middle and inner mirror modules are shown, top to bottom. Les sons learned in one radius are transferred to the others.

The mirror modules are tightly packed into the ATHENA Mirror Assembly structure, and require high precis ion with tolerances of few microns and arc seconds
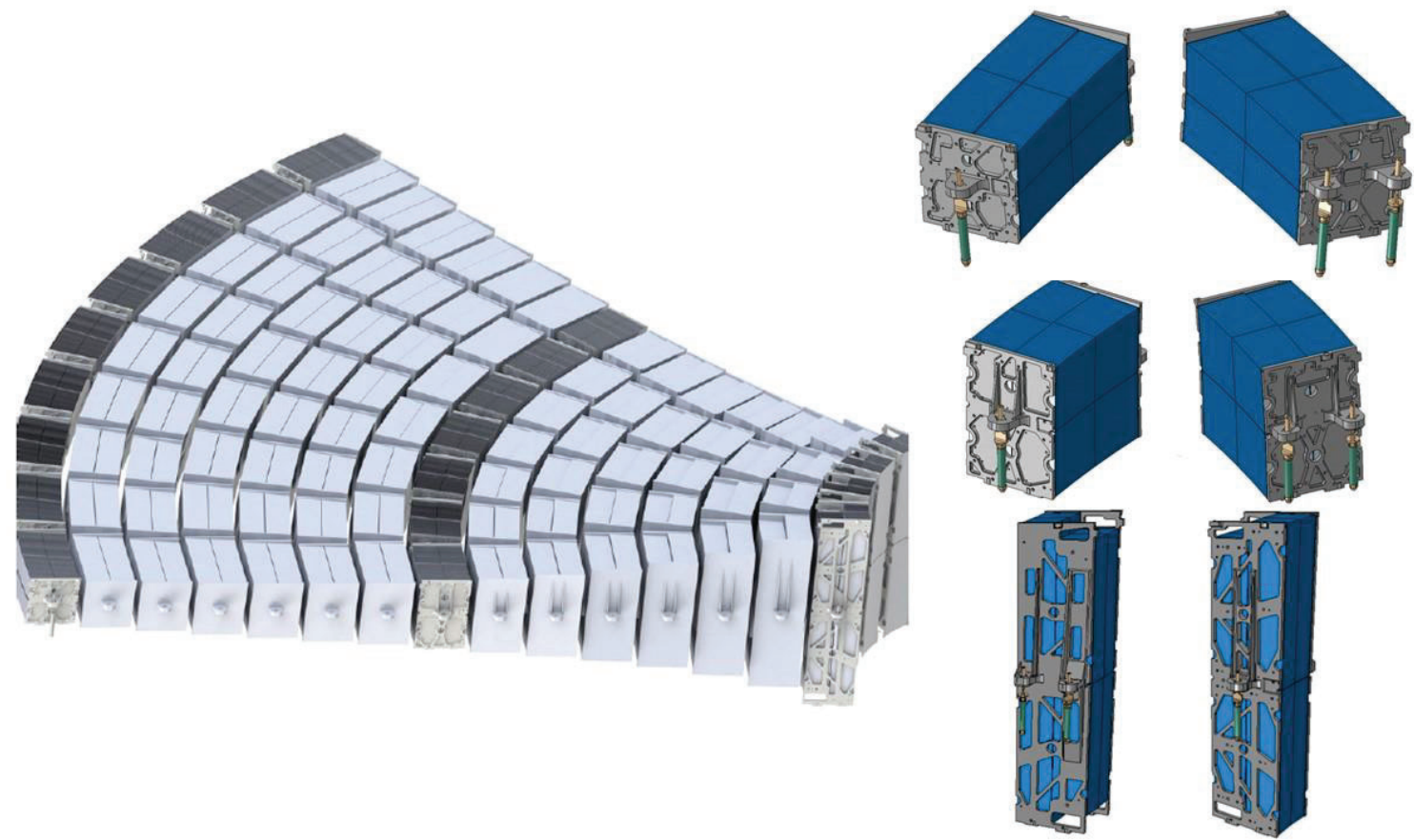

Figure 7: The ATHENA Mirror Assembly is populated by 600 carefully co-aligned mirror modules. Currently mirror modules are being developed for three representative radii: outer-most, middle and inner-most (right side of the image, top to bottom). A dedicated AIT process had to be developed to ensure the required speed and accuracy can be obtained. To demonstrate the production of the AT HENA Mirror Assembly structure activities have started with both potential primes for the implementation of ATHENA. 60 degree sectors will be initially produced, following by the complete full size demonstrator models.

The ATHENA Mirror Assembly has to be positioned on the ATHENA spacecraft with respect to the focal plane instruments. Using a hexapod system the Mirror Assembly can be tilted to focus onto one of the two focal plane instruments (figure 8 top right). A prototype of this hexapod has been built and is currently underg oing testing and verification (figure 8, bottomright).

A second hexapod systemis used during the launch sequence. This hexapod system (launch lock) is later releas ed from the Mirror Assembly, once in orbit and before commencing in-orbit commis sioning and observations. A damping system is required to ensure the optics is sufficiently protected from the mechanical shocks occurring during launch. A prototype of a bipod for such a systemis shown in figure 8 , on the left. 

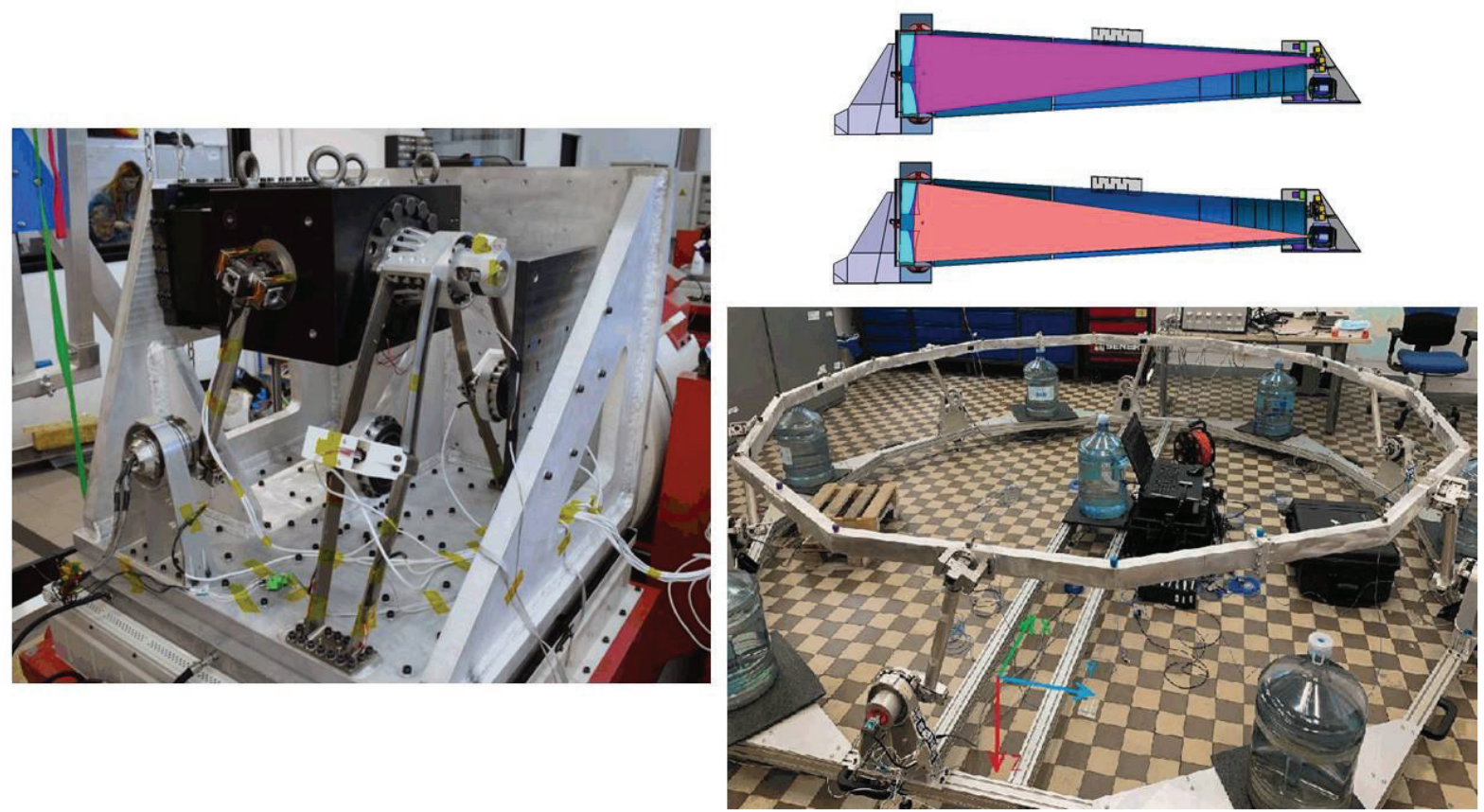

Figure 8: The ATHENA focal plane instruments are of such complexity and size, that the classical approach of moving the detectors in and out of the focal position is no longer possible. Therefore it is the optics which has to be moved in order to select the active detector instrument. The ATHENA Mirror Assembly is therefore mounted on a hexapod, which allows the tilting of the optics in the direction of the desired instrument. A further benefit of this solution is the possibility to fine tune the focus and position of the optical axis during operations in orbit. Technology activities are demonstrating the implementation of the hex ap od (right) and the launch support and damping system (left).

\section{DEFINING THE TELESCOPE GEOMETRY FOR FLIGHT}

The maturation of the optics technologies for ATHENA is running in tandem with the systemstudies. During 2020 there has been a major effort amongst all stakeholders to definea consistent geometry. This effort resulted in the first very detailed and consistent definition of the reference telescope design being agreed by everyone which will now being assumed for all the developments (fromplate level to mirror as sembly level).

The main parameters of the reference telescope design are the following (see figure 9):

- $\quad$ Focal length $=12 \mathrm{~m}$

- $487 \mathrm{~mm}<$ Opticaldiameter $<2512 \mathrm{~mm}$

- 6 sectors (i.e. Mirror Assembly with 6 fold symmetry -5 spokes)

- $100 \mathrm{MM}$ per sector (total of $600 \mathrm{MMs}$ )

- 15 rows

- 4 stacks per SPO mirror module (2 primary and 2 secondary in double reflection)

- 38 plates perstack (36 of which contributing as effectivearea) perstack

- Reference plate at the middle of the stack (plate 19)

- Plate membrane thickness of 110 microns and rib pitch of $2.3 \mathrm{~mm}$

- Wolter-Schwarzschild (W-S) geometry, where the kink of each reference plate of each stack follows the W-S sphere (i.e. is placed at a distance of $12-\mathrm{m}$ from the detector) 
- $\quad$ Plate length fixed per stack and defined for the optimal grazing angle of the reference plate per stack(resulting in stacks of different length in a MM)

- $\quad$ All plates wedged to ensure confocality

- Plate widths fixed per MM and optimis ed per row (ens uring technological limits, accommodation of nece ss ary mirror structure, heaters, dynamic and thermal distortions, etc...)
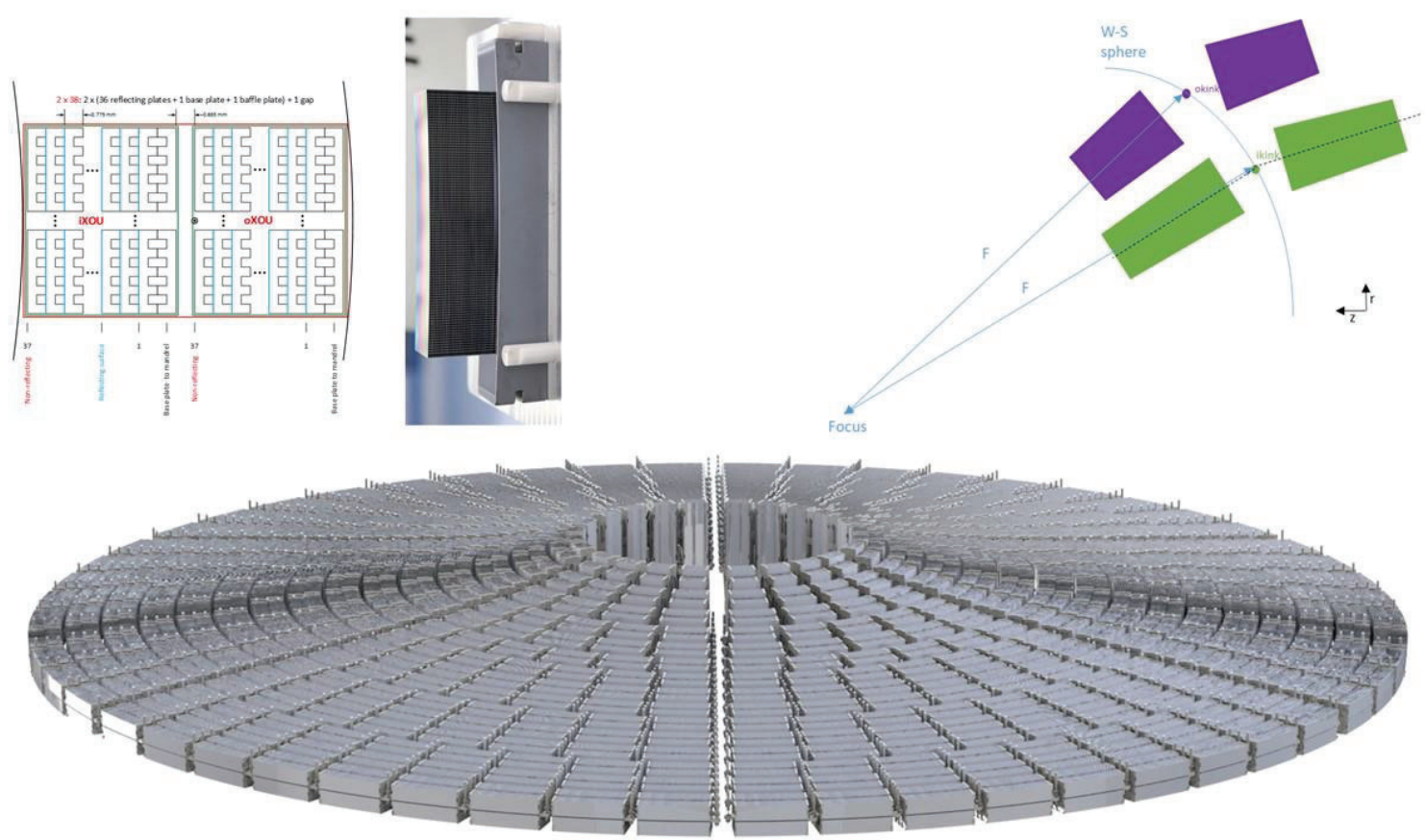

Figure 9: Definition of the AT HENA reference telescope design. Top left: Plate arrangement per stack - 38 plates per st ack (36 reflective + non-reflective baseplate + non reflective top plate). Top right: Stack arrangement on the aperture. Reference plate of each stack follows the Wolter Schwarzschild sphere (12-m from the detector), plate length fixed per stack for optimal grazing angle of reference plate leading to different stack lengths along the aperture; each MM has 4 stacks (2 primary and 2 seco nda ry). Bottom: Complete Mirror Assembly with 600 MMs arranged in the 6 identical sectors and 15 rows.

The result was the definition of all the geometric parameters of each MM and even each plate which now ensures that all mathematical models from the SPO technology activities are consistent with the Mirror Assembly Module Demonstrator and other system level activities.

The new reference telescope design also allowed some performance gains by reducing the amount of MMs (in cre a sing open area ratio) while guaranteeing that all technological limits, structural and accommodation constraints were still met. This gain was estimated to be $12 \%$ for $1 \mathrm{keV}$ and $16 \%$ for $7 \mathrm{keV}$ effective areas.

\section{ATHENA AIT AND TEST FACILITIES}

In order to ens ure the timely availability of the ATHENA mirror integration facility, the design and construction of the AIT facility has already started at the premises of Medialario near Milano, Italy. Figure 10 shows a computer ren dering of the facility. On the right is the UV Vertical Optical Beam facility (UVOB), extending about 6 m underground and about $17 \mathrm{~m}$ above. 
The UV collimator system on the bottomconsists of a $2.6 \mathrm{~m}$ diameter Zerodur mirror, currently in the final stages of polishing at Opteon in Finland, which will be used to generate a $2.6 \mathrm{~m}$ diameter UV beam, collimated to better that 1 ", pointing exactly vertically (to a fraction of arc second). This UV beamis used to position the mirror modules before fixing themto the telescope structure.

A pers on is shown for scale next to the ATHENA Mirror Assembly, preparing the integration of the next SPO mirror module (figure 10, top right). The mirror module integration process its elf is remotely controlled, ensurin g cle anlin ess and repeatability. The AIT facility is expected to be completed before the ATHENA MAR.

Co-located with the AIT facility is the Vertical X-ray Test Facility VERT-X [39] (figure 10, left). The concept of this facility has been studied in detail and its feasibility demonstrated. Thekey elements of VERT-X are the X-ray collimated beam source, a system consisting of a micro-focus X-ray source and an Wolter-I mirror, and a positioning system moving this source to cover the complete area of the ATHENA Mirror Assembly. The VERT -X facility allows the horizontal accommodation of the ATHENA Mirror Ass embly (i.e. vertical optical axis), minimising gravity induced dis tortions. Regular X-ray verifications are expected to be conducted during the process of populating the A THENA Mirror Assembly with the 600 SPO mirror modules.

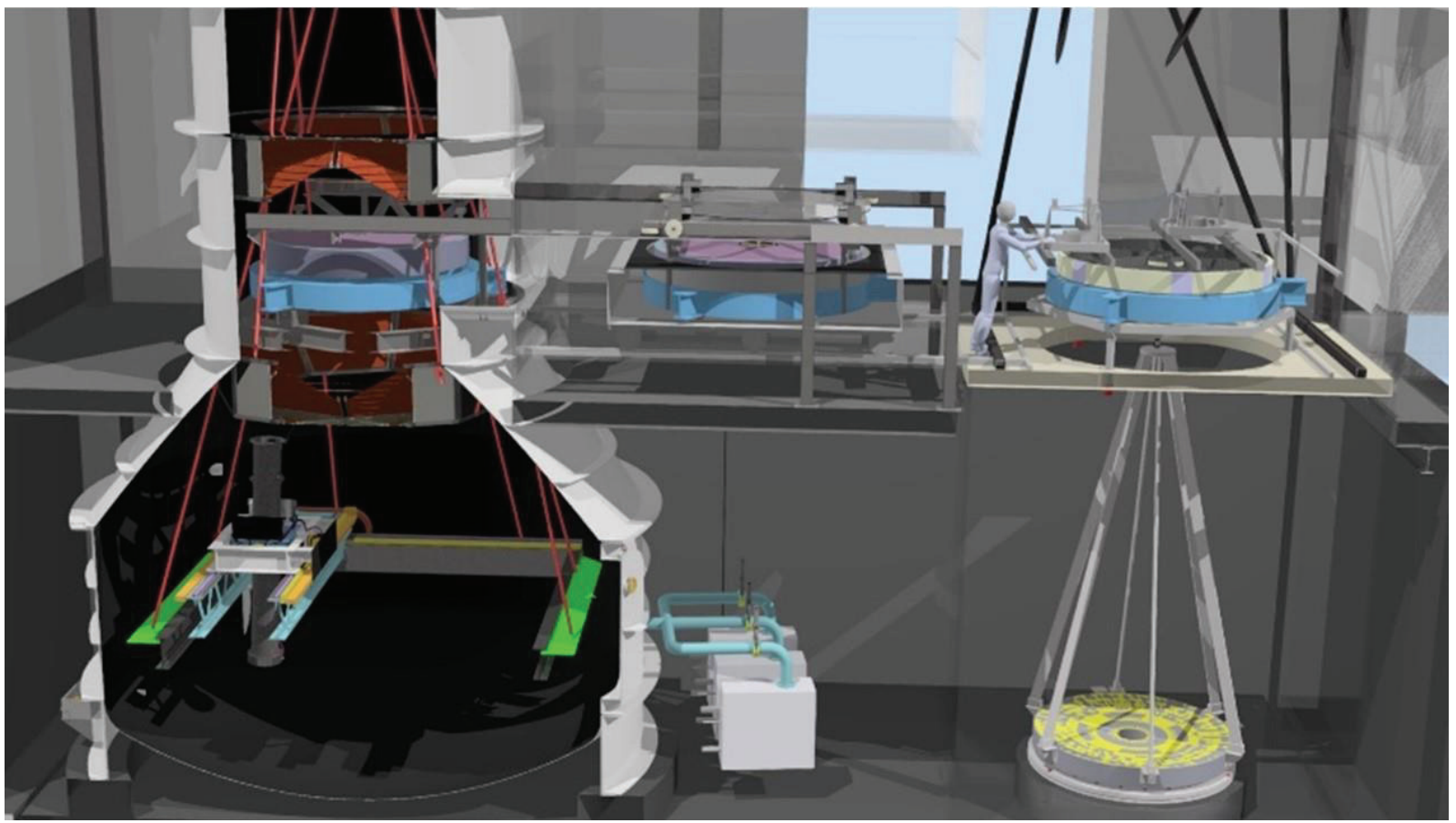

Figure 10: The size of the ATHENA optics requires the development of new AIT and X-ray test facilities. Co-location of these facilities to the extent possible is mandatory, in order to minimize the logistic complexity and cost for the ATHENA implementation. A dedicated AIT facility is being built by Medialario in Italy, which will also accommodate the Vertical X-ray Test Facility VERT -X. As shown on the right, the AIT facility consists of a large UV collimator (2.6 m diameter!), producing a vertical UV beam which is used to guide the integration of the 600 mirror modules into the ATHENA Mirror Assembly. The VERT -X facility, shown on the left, will produce a vertical collimatedX-ray beam, which will be able to scan the complete area of the AT HENA Mirror Assembly. Key elements of this facility are being implemented already. 
The large number of facilities required for the production and verification of the ATHENA optics requires adequate advance planning and implementation. As illustrated in figure 11, next to the A IT and VERT-X facilities, other major installations will be used in the implementation phase, and all are being currently prepared.

The ass embly of the SPO mirror modules requires a highly collimated X-ray beam, and two facilities will be available. BESSY-II (figure 11 top right) in Berlin, Germany, is already in use for the SPO development programme, with two dedicated beamlines being available. Further two beamlines are expected to be implemented after MAR. Since synchrotron facilities are very complexand can incur shut-downs, a further beamline is currently being implemented at the ALBA synchrotron facility (figure 11 bottomleft) near Barcelona, Spain .

The Panter facility [40] (figure 11 mid left) operated by the Max Plack Gesellschaft near Munich in Germany will continue to be used as a classic test facility with a long beamline of $130 \mathrm{~m}$ providing large area sampling.

A new type of beamline is being built by the Osservatorio Astronomico di Brera (OAB) near Milano in Italy. This facility, BEaTriX [41] (figure 11 mid right), will provide a collimated X-ray beam of sufficient size to illuminate a complete SPO mirror module, and is intended to be used as a mirror acceptance testing and calibration facility.

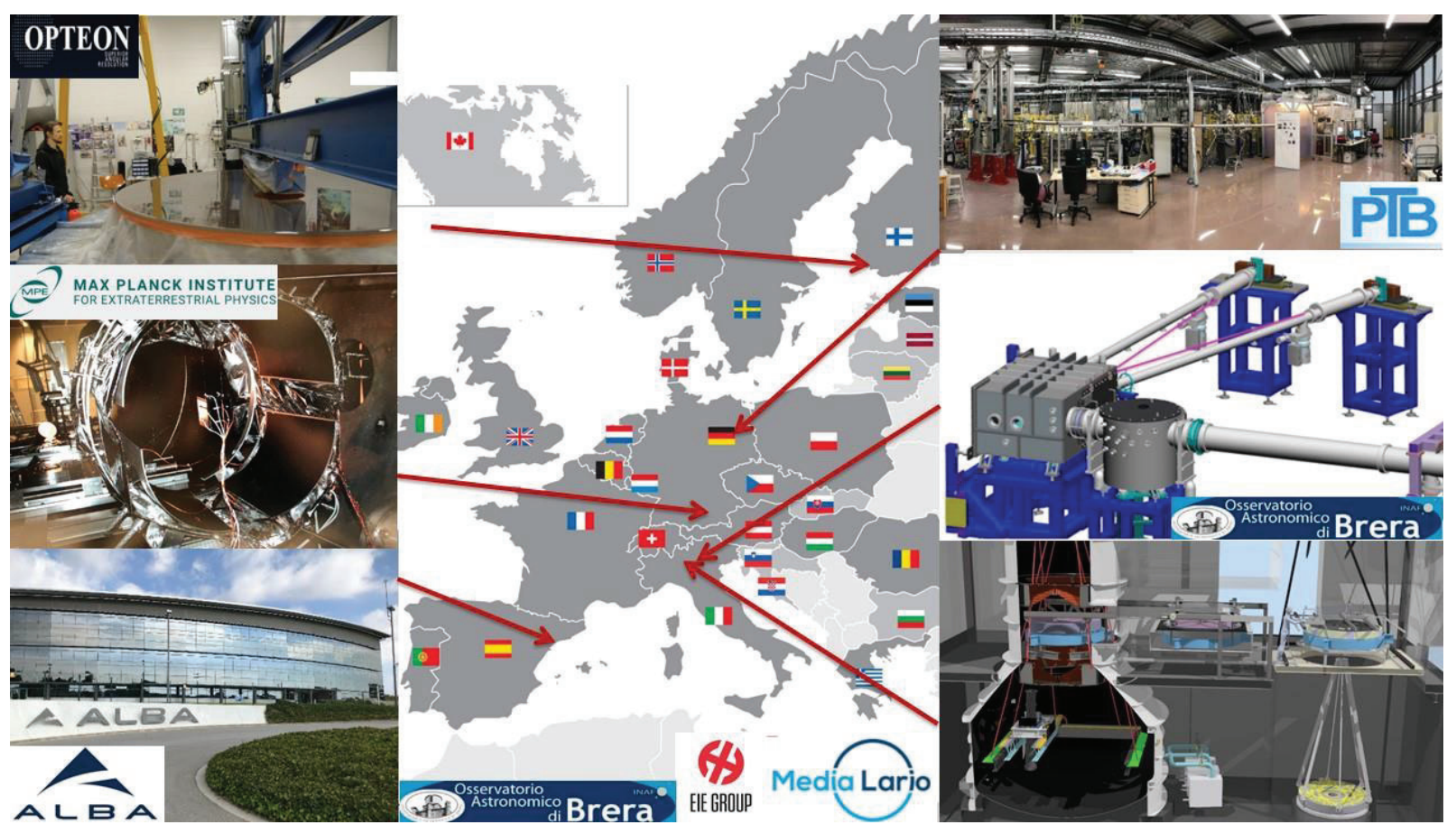

Figure 11: A large number of facilities are required for implementing the ATHENA telescope optics and to verify it's performance. The facilities are located across Europe and consist of synchrotron beamlines required for the assembly of mirro $\mathrm{r}$ modules (BESSY-II in Berlin, Germany, and ALBA Synchrotron near Barcelona, Spain), mirror module acceptance and calibration facilities (Panter near Munich, Germany, and BEaTriX near Milano, Italy), the AIT and VERT -X facilities (near Milano, Italy) with the UV collimator (produced by Opteon in Finland). In addition (not shown) the NASA XRCF facility in Huntville, Alabama, USA, is being prepared for testing the ATHENA optics. 


\section{CONCLUSION}

ATHENA is the second Large Class Science mission of ESA, and is enabled by a no vel type pfX-ray optics. The Silicon Pore Optics (SPO) technology combines low mass, good angular resolution and large effective area in a way no other existing X-ray optics technology able to operate in space has achieved to date.

The SPO technology is being developed in a holistic approach, addressing the performance and the programmatic (schedule and cost) aspects related to the mass production required for ATHENA, as 600 mirror modu les have to be produced in about 2 years.

A coordinated technology plan is being implemented, being fully funded and led by ESA and involving ind ustrial and institutional players. Existing expertise, commercial equipment, processes and components are being combined and brought to fruition to develop and manufacture the largest X-ray optics ever flown in space.

Benefiting fromimmense investments made by the semiconductor industry and spinning in technologies develop ed for the ground sector, it became possible to satis fy the needs of ATHENA. The running developments will proceed until the ATHENA Mission Adoption Review, currently expected in 2022, and will evolve into the implementation phase, aiming at a launch in the early 2030 s.

With a modular approach, the ATHENA optics of about $2.5 \mathrm{~m}$ in diameter is populated with 600 mirror modules, which have to be accurately aligned. A dedicated integration process was developed and the facility required for the full size ATHENA optics is being implemented.

The verification and calibration of the large ATHENA optics poses its own challenges and required new test facilities, which are also being developed and implemented. Coordination with existing NASA facilities, in particular the XRCF, is ongoing in addition.

\section{ACKNOWLEDGEMENTS}

The authors would like to express their appreciation for the great collaborative spirit in the joint endea vour to develop and produce the optics for the ATHENA mission.

\section{REFERENCES}

[1] K. Nandra, "Athena: Exploring the Hot and Energetic Universe", Proc. American Astronomical Society HEAD meeting (\#14), (2014).

[2] Meidinger, N., et al, "The Wide Field Imager instrument for Athena", Proc. SPIE 9905, Space Telescopes and Instrumentation 2016: Ultraviolet to Gamma Ray, 99052A (18 July 2016)

[3] Barret, D., et al, "The ATHENA X-ray Integral Field Unit (X-IFU), Proc. SPIE 10699, Space Telescopes and Instrumentation 2018: Ultraviolet to Gamma Ray, 106991G(31 July 2018)

[4] Ryo Iizuka, et al, "Ground-based x-ray calibration of the Astro-H/Hitomi soft x-ray telescopes", J. Astron. Telesc. Instrum. Syst. 4(1), 011213 (2018)

[5] William W. Craig, et al., "Fabrication of the NuST AR Flight Optics", Proc. of SPIE Vol. 8147, 81470H (2011)

[6] Jansen, F., et al, "XMM-Newton observatory”, Astronomy and Astrophysics, vol. 365, no. 1, pp. L1-L6 (2001)

[7] O’Dell, S.L. and M. C. Weisskopf "“Advanced X-ray astrophysics facility (AXAF): Calibration overview”, Proc. SPIE 3444 (1998) and references therein

[8] Beijersbergen, M. et al., "Silicon pore optics: novel lightweight high-resolution X-ray optics developed for XEUS", Proc. SPIE 5488, 868-874 (2004).

[9] Bavdaz, M., et al, United States Patent US7,321,127 B2 (2008)

[10] Wallace, K. et al, "Silicon pore optics development", Proc. SPIE 7437, 7437 (2009)

[11] Bavdaz, M., et al, "X-Ray Pore Optics Technologies and Their Application in Space Telescopes", Hindawi Publishing Corporation X-Ray Optics and Instrumentation Volume 2010, Article ID 295095 (2010)

[12] Collon, M. J. et al, “Silicon Pore X-ray Optics for IXO”, Proc. SPIE 7732, 77321 F (2010) 
[13] Bavdaz, M., “ESA optics technology preparation for IXO”, Proc. SPIE 7732, 77321E (2010)

[14] Willingale, R. et al, "Science requirements and optimization of the silicon pore optics design for the AT HENA mirror", Proc. of SPIE Vol. 9144, 91442E (2014)

[15] Landgraf, B., et al, "Environmental testing of the ATHENA mirror modules", Proc. of SPIE Vol. 10399, 103990G-1 (2017)

[16] Fransen, S. et al, "Prediction of the mechanical environments of the load critical elements of the ATHENA spacecraft", Proc. 15th European Conference on Spacecraft Structures, Materials and Environmental Testing (2018)

[17] Collon, M., et al, "Silicon Pore Optics Mirror Module Production and Testing”, Proc. of SPIE Vol. 11180, 1118023 (2018)

[18] Wille, E., et al., "Silicon pore optics manufacturing plan and schedule for ATHENA", Proc. SPIE, 106993L (2018)

[19] Collon, M., et al., "Status of the silicon pore optics Technology”, Proc. SPIE, 111190L (2019)

[20] Barriere, N., et al, "Assembly of confocal silicon pore optic mirror modules for Athena”, Proc. SPIE, 111190J (2019)

[21] Keek, L., et al, "Stacking of mirrors for silicon pore Optics", Proc. SPIE, $111190 \mathrm{H}$ (2019)

[22] Bavdaz, M., et al, “Optics developments for ATHENA”, Proc. SPIE, 111190D (2019)

[23] Vacanti, G., et al, "X-ray testing of silicon pore optics”, Proc. SPIE, 111190I (2019)

[24] Spiga, D., et al, "Simulation and modelling of silicon pore optics for the ATHENA x-ray telescope", Proc. SPIE. 9905, $990550(2016)$

[25] Vacanti, G., et al, “Predicting Silicon Pore Optics”, Proc. of SPIE Vol. 10399, 103990M-1 (2017)

[26] Fioretti, V., et al, "The Geant4 mass model of the ATHENA Silicon Pore Optics and its effect on soft proton scat tering", Proc. of SPIE Vol. 10699, 106993J(2018)

[27] Della Monica Ferreira, D. et al., "Design, development, and performance of X-ray mirror coatings for the ATHENA mission", Proc. of SPIE Vol. 10399, 1039918-1 (2017)

[28] Massahi, S., et al, "Industrialization of the mirror plate coatings for the ATHENA mission", Proc. of SPIE Vol. 10399, 103991W-1 (2017)

[29] Della Monica Ferreira, D. et al., "Performance and Stability of Mirror Coatings for the ATHENA Mission", Proc. of SP IE Vol. 10699, 106993K (2018)

[30] Massahi, S., et al, "Balancing of residual stress in thin film iridium by utilizing chromium as an underlayer", Proc. of SPIE Vol. 11444, 114444N (2020)

[31] Hisamitsu Awaki, et al, "Measuring the atomic scattering factors near the iridium L-edges for the Athena silicon pore optics reflector”, J. Astron. T elesc. Instrum. Syst. 014001-1 Jan-Mar 2021 • Vol. 7(1) (2021)

[32] Valsecchi, G., et al, “ATHENA Telescope: alignment and integration of SPO mirror modules”, Proc. of SPIE Vol. 11180 , $111801 \mathrm{~N}(2018)$

[33] Vernani, D., et al, "Integration of the ATHENA mirror modules: development status of the indirect and direct X-ray methods", Proc. of SPIE Vol. 10699, 1069910 (2018)

[34] Valsecchi, G., et al, "Integration facility for the ATHENA X-Ray Telescope”, Proc. SPIE, 111190M (2019)

[35] Ayre, M., et al, “ATHENA: phase A study status and optics/instrument accommodation”, Proc. SPIE 11119, Optics for EUV, X-Ray, and Gamma-Ray Astronomy IX, 111190R(2019)

[36] ISO, "Space systems - Definition of the Technology Readiness Levels (T RLs) and their criteria of assessment", ISO 16290, The International Organization for Standardization (2013)

[37] Krumrey, M. et al, “New X-ray parallel beam facility XPBF 2.0 for the characterization of silicon pore optics”, Proc. SPIE. 9905, 99055N. (2016)

[38] Handick, Evelyn, et al, "Upgrade of the x-ray parallel beam facility XPBF 2.0 for characterization of silicon pore opt ics", Proc. of SPIE Vol. 11444, 114444G(2020)

[39] Moretti, A., et al, "VERT-X: VERT ical X-ray raster-scan facility for ATHENA calibration. The concept design", Proc. SPIE $11119,1111900(2019)$

[40] Burwitz, V., et al, "X-ray Testing at PANTER of Optics for the AT HENA and Arcus Missions", Proc. of SPIE Vol. 11180 , 1118024 (2018)

[41] Salmaso, B., et al, "BEaTriX (Beam Expander Testing X-ray facility) for testing AT HENA's SPO modules: advanc em ent status", Proc. of SPIE Vol. 11180,1118026(2018) 